\title{
Algunas consideraciones sobre el uso de técnicas fractales en el análisis del mercado accionario bursátil mexicano
}

\begin{abstract}
Alfredo Díaz Mata*
Resumen

En este trabajo se aportan pruebas de que los rendimientos de las acciones que cotizan en la Bolsa Mexicana de Valores, así como también su índice de precios, no cumplen con dos de los supuestos en los que se basa buena parte de la teoría de cartera moderna. Estos supuestos son que las series tienen distribución normal y que los rendimientos sucesivos son independientes.

Se revisan, por un lado, los planteamientos de la teoría del paseo aleatorio y los de la hipótesis del mercado eficiente y, por otra parte, el supuesto de que la distribución de esos rendimientos es normal para mostrar que, en ambos casos, no se cumplen los supuestos con series del mercado bursátil mexicano.

Después se ve que las series de tiempo de rendimientos tienen características fractales a las que se les puede aplicar una técnica conocida como "análisis de rangos redimensionados”, como una técnica adicional, fractal, para tratar de probar que sí hay dependencia entre rendimientos sucesivos de los títulos.
\end{abstract}

Se señala la necesidad de aplicar modelos que prescindan de esos dos supuestos para lograr mejores descripciones de la realidad y mejores pronósticos.

Palabras clave: fractales, análisis de rangos redimensionados, bolsa de valores.

Fecha de recepción: 28-09-2006

Fecha de aceptación: 05-07-2007

Some Consideration about Using Fractal Techniques in the Mexican Stock Exchange

Abstract

Proof is offered in this paper that the returns of the stocks negotiated in the Mexican Stock Exchange, and its own index, do not comply with two of the assumptions on which

*Investigador de la División de Investigación de la Facultad de Contaduría y Administración, UNAM. Correo electrónico: adiazm@correo.fca.unam.mx

No. 224, enero-abril 2008:35-57 
a good deal of the modern portfolio theory is based. These assumptions are that the series are normally distributed and that the successive returns are independent.

The random walk theory and the efficient market hypothesis are reviewed, together with the normality of the stock market returns, to show that in both cases, the suppositions do not apply to price series of the Mexican stock market.

Also, it is shown how the time series of stock returns have fractal characteristics to which a method known as "rescaled range analysis" can be applied, as a fractal and additional technique, in order to prove that, indeed, there is dependence among the successive stock returns.

It is pointed out that it is necessary to apply models that do not include these suppositions so as to obtain better descriptions of reality and better price forecasts.

Key words: fractals, rescaled range analysis, Mexican stock market.

\section{Introducción}

- 1 presente trabajo forma parte de un estudio más amplio y de largo plazo desaCrrollado por el autor de estas líneas y que inició propiamente con la publicación en 1996 de un artículo titulado "Caos, fractales, mercado de valores y triceversa" y que ha continuado desde entonces con la publicación, en el mismo medio que el anterior, de varios artículos más sobre mercados financieros, mercado bursátil, teoría de cartera y análisis bursátil de acciones y con la elaboración de una tesis de maestría y una de doctorado, que incluyeron estos mismos temas, pero que versaron, principalmente, sobre el tema de análisis técnico de acciones bursátiles.

Se retoma ahora el análisis fractal del mercado accionario mexicano, que está íntimamente relacionado con el análisis técnico en cuanto que ambos se basan primordialmente en el estudio de los precios de los valores que cotizan en bolsa. Esta exploración se basa, sobre todo, en resultados obtenidos por quien esto escribe y también en los planteamientos de un autor, Edgar E. Peters ${ }^{2}$, quien propone un análisis fractal de los mercados financieros partiendo de las premisas de que la hipótesis de los mercados eficientes (HME) y, junto con ésta, la teoría del paseo aleatorio (TPA) no son aplicables a estos mercados porque no se cumplen varios de los supuestos en los que se basan esa hipótesis y esa teoría y entre los cuales es posible que los prin-

\footnotetext{
${ }^{1}$ Díaz Mata, Alfredo, "Caos, fractales, mercados de valores y triceversa”, Contaduría y Administración, 1996.

${ }^{2}$ Peters, Edgar E., Chaos and Order in the Capital Markets, 1991 y Fractal Market Analysis, 1994. 
Algunas consideraciones sobre el uso de técnicas fractales

en el análisis del mercado accionario bursátil mexicano

cipales supuestos sean la independencia y la distribución normal de los rendimientos sucesivos de instrumentos bursátiles.

De igual manera, en este trabajo se hace un análisis, con técnicas que se pueden calificar como "fractales", en el que se cuestiona ese supuesto de independencia de los rendimientos sucesivos de instrumentos bursátiles, utilizando para ello precios y rendimientos del Índice de Precios y Cotizaciones (IPC) de la Bolsa Mexicana de Valores y de cuatro acciones que cotizan en esta bolsa. Por otro lado, se presentan algunas consideraciones sobre la supuesta normalidad de esos rendimientos.

El trabajo está organizado en varias secciones. En la primera, la siguiente, se repasan los principales conceptos relacionados con la hipótesis de los mercados eficientes y la teoría del paseo aleatorio, directamente relacionadas con el supuesto de independencia de los rendimientos sucesivos de valores bursátiles. En la segunda sección se resumen varios ejemplos que ilustran casos de obtención de rendimientos por encima del IPC y que contradicen a la HME y a la TPA. En la tercera sección se analiza el comportamiento del IPC, como ejemplo de que esos rendimientos bursátiles no tienen distribución normal y en la sección cuarta se exponen nociones básicas sobre el análisis técnico y los fractales, de donde se desprende la quinta sección, que se ocupa del exponente $\mathrm{H}$ de Hurst que es un mecanismo que se ha propuesto para probar la dependencia o independencia de los rendimientos bursátiles, el otro supuesto que se quiere evaluar en este trabajo para el mercado mexicano. El documento termina con comentarios finales y la bibliografía.

\section{La hipótesis de los mercados eficientes y la teoría del paseo aleatorio}

La hipótesis de los mercados eficientes fue propuesta formalmente por Eugene F. Fama y plantea que

Un mercado de capitales eficiente es un mercado que es eficiente en el procesamiento de la información. Los precios observados de los valores, en cualquier momento, están basados en la "correcta" evaluación de toda la información disponible en ese momento. En un mercado eficiente, los precios "reflejan totalmente" la información disponible. ${ }^{3}$

Este planteamiento implica, entre otras cosas, que no se puede prever el comportamiento de los precios de manera que permita obtener rendimientos superiores a los

${ }^{3}$ Fama, Eugene F., Foundations of Finance, 1976, p. 133. 
que se reflejan en un índice accionario representativo del mercado, como sería el IPC en el caso mexicano. Esta hipótesis tiene tres formas ${ }^{4}$ :

La forma "débil" sostiene que todos los precios y datos pasados sobre el mercado están completamente reflejados en los precios de los títulos, lo cual implica que el análisis técnico, el análisis bursátil que se basa en el estudio de los precios de los títulos, no tiene ninguna utilidad.

La forma "semifuerte" sostiene que toda la información públicamente disponible está completamente reflejada en los precios de los valores, lo cual implica que el análisis fundamental, el análisis bursátil que se basa en el estudio de los fundamentos financieros de las empresas, de acuerdo con sus estados financieros y en el estudio de su entorno micro y macroeconómico, no tiene ninguna utilidad.

La forma "fuerte" sostiene que toda la información está totalmente reflejada en los precios de los títulos, incluyendo la información privilegiada, lo cual implica que tampoco este tipo de información tiene utilidad alguna.

Aunque existen numerosos estudios que dan apoyo a hipótesis de los mercados eficientes, también se han documentado ciertas "anomalías técnicas" y "anomalías del mercado accionario" que muestran que los mercados accionarios no son ni totalmente eficientes ni totalmente ineficientes lo cual, a su vez, señala que, bajo ciertas condiciones, sí es posible prever movimientos futuros de los precios de las acciones.

Por su parte, los fundamentos de la teoría del paseo aleatorio provienen de marzo de 1900, con la presentación que hizo Louis Bachelier de su tesis de doctorado titulada "La teoría de la especulación", revisada y autorizada por un comité en el que participó Henri Poincaré, en los siguientes términos: "En resumen, somos de la opinión de que existe razón para autorizar al Sr. Bachelier para que imprima y presente su tesis." $"$

Bachelier, estudiando el comportamiento de los precios en el mercado bursátil francés, concluye que "la esperanza matemática del especulador es nula" lo cual implica, precisamente, que no es posible anticipar los movimientos de los precios para,

${ }^{4}$ Adaptado de Ross, S., Westerfield, R. y Jordan, B., Fundamentos de finanzas corporativas, 1996, p. 367.

${ }^{5} \mathrm{http}: / /$ www.investorhome.com/anomtec.htm

${ }^{6}$ Idem.

${ }^{7}$ Bachelier, Louis, Théorie de la spéculation, 1900.

${ }^{8} \mathrm{http}: / /$ www.stochastik.uni-freiburg.de/bfsweb/LBachelier/mafi092XX.pdf, p. 349.

${ }^{9}$ Bachelier, op. cit., p. 34. Traducción propia. 
Algunas consideraciones sobre el uso de técnicas fractales

en el análisis del mercado accionario bursátil mexicano

con ello, obtener utilidades por encima de los rendimientos promedio del mercado porque, a la larga, el especulador ni gana ni pierde con respecto a esos rendimientos promedio del mercado.

Esta autora comenta en la introducción de su tesis que "las influencias que determinan los movimientos de la bolsa son innumerables e incluyen eventos pasados, actuales e incluso algunos previsibles" por lo que considera que "el cálculo de probabilidades indudablemente no podrá jamás ser aplicado a los movimientos de los niveles y que la dinámica de la bolsa no será jamás una ciencia exacta." 10

Y cita Bachelier al final de su trabajo: "Es posible que un comentario final no resulte inútil. $\mathrm{Si}$, a la vista de las diversas cuestiones tratadas en este estudio, he comparado los resultados de la observación con los de la teoría, no ha sido para verificar las fórmulas establecidas por los métodos matemáticos, sino para mostrar solamente que el mercado, sin saberlo, obedece a una ley que lo domina: la ley de la probabilidad". ${ }^{11}$ Es decir, el azar.

También Eugene Fama describe una "teoría del paseo aleatorio"12 que se basa en dos hipótesis. La primera de ellas, y la más importante, plantea que los cambios sucesivos en los precios de las acciones bursátiles son independientes y la segunda hipótesis afirma que los cambios en los precios se ajustan a alguna distribución de probabilidades.

La primera hipótesis implica que no es posible pronosticar el cambio en los precios con base en los cambios anteriores, precisamente porque son aleatorios.

Por su parte, Burton G. Malkiel plantea lo siguiente:

Un paseo aleatorio es aquel en el que los pasos o la dirección a seguir en el futuro no se pueden predecir a partir de las acciones anteriores. Cuando este término se aplica al mercado de valores, quiere decir que no se pueden predecir a corto plazo los cambios en la cotización de las acciones. ${ }^{13}$

Sin embargo, el mismo autor señala que "Llego a la conclusión de que, aunque los anuncios sobre la muerte de la teoría del mercado eficiente son muy exagerados,

\footnotetext{
${ }^{10}$ Ibídem, p. 21.

${ }^{11}$ Ibídem, p. 86.

${ }^{12}$ Fama, Eugene, “The Behavior of Stock Market Prices", Journal of Business, 1965, pp. 34-105.

${ }^{13}$ Malkiel, Burton G., Un paseo aleatorio por Wall Street, España, 1992, p. 24.
} 
parecen existir algunas técnicas de selección de valores que pueden inclinar las probabilidades de éxito a favor del inversor particular". ${ }^{14}$

\section{Rendimientos por encima del IPC}

Como puede verse de lo anterior, la hipótesis de los mercados eficientes y la teoría del paseo aleatorio están estrechamente relacionadas y, en cuanto concierne a este trabajo, su principal implicación es que ambas plantean que no es posible utilizar información previa (como los historiales de precios en el análisis técnico o los datos financieros y macroeconómicos de las empresas en el análisis fundamental) para hacer pronósticos de los precios de las acciones bursátiles. Sin embargo, aparte de las "anomalías" anotadas antes, existen diversas evidencias de que sí es posible hacer pronósticos exitosos de esos precios con técnicas de ambos tipos de análisis bursátil.

En la ponencia presentada en el X Foro de Investigación, que se llevó a cabo en la Facultad de Contaduría y Administración de la UNAM, en septiembre de 2005, el autor de este trabajo presentó una exposición titulada "El análisis bursátil, la hipótesis de los mercados eficientes y la teoría del paseo aleatorio", en la que se presentó un conjunto de ejemplos que contradicen a la HME. Se resumen enseguida estos ejemplos:

- Una conclusión de Francisco López Herrera: “Mediante las pruebas realizadas para el periodo de referencia, no se puede afirmar que los rendimientos del IPC de la Bolsa Mexicana de Valores sigan una caminata aleatoria." 15.

- Un artículo del afamado (y acaudalado) financiero estadunidense Warren E. Buffet $^{16}$ en el que da cuenta de un conjunto de inversionistas (inversionistas individuales y empresas de inversión) que le han ganado al índice Standard \& Poor's de la Bolsa de Valores de Nueva York, "año tras año", y que son inversionistas conocidos por él y de fama pública.

- Un ejercicio con 46 sociedades de inversión comunes (SIC) mexicanas, de las cuales diversos números de ellas mostraron un rendimiento superior al del Índice de Precios y Cotizaciones (IPC) de la Bolsa Mexicana de Valores (BMV) en periodos de diversa duración entre los 60 meses que transcurrieron de junio de 2000 a mayo de 2005.

${ }^{14}$ Ibídem, p. 16.

${ }^{15}$ López Herrera, Francisco, “Análisis de la eficiencia del mercado accionario mexicano”, Contaduría y Administración, 1998, pp. 82-83.

${ }^{16}$ Buffet, Warren E., "The Superinvestors of Grahamanddoddsville", Hermes de la Columbia Business School, artículo reimpreso en http://www.sandmansplace.com/Superinvestors_Graham_Dodd.html, y que fue originalmente elaborado con base en un discurso presentado por Buffet en esa Columbia Business School el 17 de mayo de 1984, en el curso de un seminario organizado para celebrar el quincuagésimo aniversario de la publicación del libro Security Análisis, de Benjamín Graham y David Dodd. 
Algunas consideraciones sobre el uso de técnicas fractales

en el análisis del mercado accionario bursátil mexicano

- Se presentaron los alentadores y positivos resultados obtenidos en inversiones reales en acciones en la Bolsa Mexicana desde enero de 2002 y hasta mediados de agosto de 2005.

- Finalmente, se realizaron pruebas de aleatoriedad para las series de los rendimientos de las 46 acciones de sociedades de inversión comunes y del IPC con las que se llega a la conclusión de que los rendimientos de éste Índice y de 10 acciones no tienen un comportamiento aleatorio.

Sin embargo, estos ejemplos de rendimientos superiores son sólo eso, ejemplos, y no muestran que se hayan encontrado mecanismos que permitan obtener sistemáticamente rendimientos superiores al promedio en el mercado accionario, a pesar de las abundantes publicaciones que pretenden ofrecer esas técnicas.

Por ello, vale la pena intentar consideraciones adicionales para tratar de llegar a ese fin; la teoría de los fractales, el caos y los sistemas dinámicos lineales parecen ofrecer una alternativa.

\section{Sobre el supuesto de normalidad de los rendimientos bursátiles}

Parte de los análisis modernos de la teoría de cartera se basan en normalidad y la distribución de los rendimientos del Índice de Precios y Cotizaciones de la Bolsa Mexicana de Valores no es normal.

Por ejemplo, en el capítulo 10 de Ross, Westerfield y Jordan ${ }^{17}$ (1995) titulado "Algunas lecciones históricas del mercado de capital" se incluye una sección sobre el análisis del comportamiento histórico de los rendimientos de diversos instrumentos del mercado de capitales de Estados Unidos, que contiene acciones, bonos y certificados de la tesorería.

Después de esta sección, en el mismo capítulo, se incluyen dos secciones más, la 10.4, titulada "Rendimientos promedio: la primera lección", en la que se resalta, básicamente, el principio financiero que afirma que a mayor riesgo se obtiene mayor rendimiento y se ponen ejemplos que ilustran que, por lo general y a largo plazo, son superiores los rendimientos promedio de las acciones que los de instrumentos de deuda como certificados de la tesorería.

En la sección siguiente, la 10.5, titulada "La variabilidad de los rendimientos: la segunda lección", incluye un apartado que lleva como encabezado "La distribución normal”, en el cual se exponen diversos ejemplos, en los que se evalúa la proba-

${ }^{17}$ Ross, Westerfield y Jordan, op. cit. 
bilidad de alcanzar cierto rendimiento con base, precisamente, en esta distribución normal. Un caso en cuestión:

[...] suponiendo que la distribución de frecuencias es normal, al menos aproximadamente, la probabilidad de que el rendimiento en un año determinado se localice en el intervalo del -8.7\% al 32.9\% (12.1\% más o menos una desviación estándar, 20.8\%) es de alrededor 2/3. ${ }^{18}$

El ejemplo anterior es uno de los muchos que se pueden encontrar sobre la interpretación que se suele hacer del comportamiento de los rendimientos bursátiles, que tienen una distribución normal o "aproximadamente normal", afirmación sobre la que se han planteado diversos cuestionamientos, como el de Benoit Mandelbrot, citado y analizado por Eugene F. Fama ${ }^{19}$, en el que se afirma que la distribución de esos rendimientos no es normal, sino que sigue a una distribución denominada distribución estable de Pareto.

Las distribuciones estables de Pareto son una familia de distribuciones definidas por cuatro parámetros. Delta, $\delta$, de ubicación o posición, gama, $\gamma$, de escala, beta, $\beta$, de sesgo, y alfa, $\alpha$, una medida de la altura de las colas de la distribución al que se denomina el exponente característico.

Por su parte, la distribución normal es un caso particular de la familia de las distribuciones estables de Pareto, en la cual $\alpha=2$, lo cual implica que la altura de las colas es, precisamente, la de la distribución normal, $\beta=0$, lo cual equivale a decir que se trata de una distribución simétrica y tanto $\gamma$ como $\delta$ pueden ser cualquier número positivo. Entonces, en el análisis que sigue se pretende ilustrar cómo es que la distribución de los rendimientos de instrumentos del mercado de capitales y, específicamente, de las acciones, tiene un comportamiento que se ajusta mejor a una distribución estable de Pareto, diferente de la normal, ya que se trata de distribuciones sesgadas, leptokúrticas (es decir, más puntiagudas), de colas más anchas (o gordas, si se quiere) y con mayor número de observaciones en ambos extremos, pero particularmente en el izquierdo.

Para evaluar estas características en el sistema bursátil mexicano, se utilizan datos de rendimientos del Índice de Precios y Cotizaciones de la Bolsa Mexicana de Valores, del 4 de noviembre de 1985 al 25 de septiembre de 2006, o casi 21 años de datos diarios de días hábiles. En primer lugar, se construyó una distribución de frecuencias de los rendimientos observados y de los esperados, que es la que se muestra en la tabla 1.

\footnotetext{
${ }^{18}$ Ibídem, p. 361

${ }^{19}$ Fama, Eugene F., "The Behavior of Stock Market Prices”, Journal of Business, 1965, pp. 33-105. 
Algunas consideraciones sobre el uso de técnicas fractales en el análisis del mercado accionario bursátil mexicano

Tabla 1

Distribución de frecuencias de rendimientos diarios del IPC de la BMV (del 4 de noviembre de 1985 al 25 de septiembre de 2006)

\begin{tabular}{|c|c|c|}
\hline Rendimiento & F observada & Frecuencia esperada \\
\hline-0.2 a menos de -0.18 & 1 & 0 \\
\hline-0.18 a menos de -0.16 & 2 & 0 \\
\hline-0.16 a menos de -0.14 & 2 & 0 \\
\hline-0.14 a menos de -0.12 & 3 & 0 \\
\hline-0.10 a menos de -0.10 & 2 & 0.002 \\
\hline-0.10 a menos de -0.08 & 6 & 0.260 \\
\hline-0.08 a menos de -0.06 & 11 & 12.618 \\
\hline-0.06 a menos de -0.04 & 57 & 207.004 \\
\hline-0.04 a menos de -0.02 & 312 & 1149.558 \\
\hline-0.02 a menos de 0.00 & 1989 & 2161.011 \\
\hline 0.00 a menos de 0.02 & 2284 & 1375.175 \\
\hline 0.02 a menos de 0.04 & 435 & 296.233 \\
\hline 0.04 a menos de 0.06 & 84 & 21.601 \\
\hline 0.06 a menos de 0.08 & 21 & 0.533 \\
\hline 0.08 a menos de 0.10 & 8 & 0.004 \\
\hline 0.10 a menos de 0.12 & 2 & 0 \\
\hline 0.12 a menos de 0.14 & 3 & 0 \\
\hline 0.14 a menos de 0.16 & 0 & 0 \\
\hline 0.16 a menos de 0.18 & 1 & 0 \\
\hline 0.18 a menos de 0.20 & 0 & 0 \\
\hline 0.20 a menos de 0.22 & 0 & 0 \\
\hline 0.22 a menos de 0.24 & 0 & 0 \\
\hline 0.24 a menos de 0.26 & 0 & 0 \\
\hline 0.26 a menos de 0.28 & 1 & 0 \\
\hline 0.28 a menos de 0.30 & 0 & 0 \\
\hline
\end{tabular}

Estos mismos datos son los que aparecen graficados en la figura 1, en donde la línea punteada ilustra las frecuencias esperadas de acuerdo con una distribución normal, con media de 0.0016544 y desviación estándar de 0.01921661 , que son los parámetros observados. 
Figura 1

Distribuciones de frecuencias observada y esperada

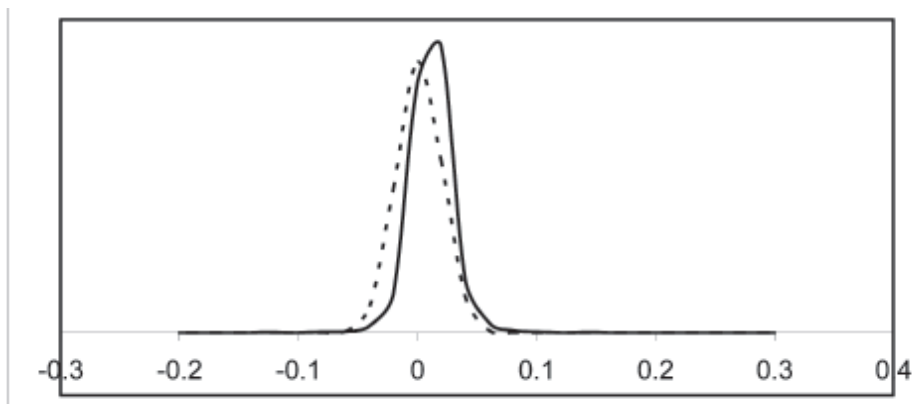

Comparando las frecuencias observadas contra las esperadas y las gráficas de ambas distribuciones que se muestran en la figura 1 se puede observar que:

La distribución de las frecuencias observadas es más puntiaguda (leptokúrtica) que la de las esperadas de acuerdo con la distribución normal.

La distribución observada tiene las colas más anchas, lo cual se puede observar en la tabla, pero también gráficamente observando que la cola del lado derecho es más alta que la del lado izquierdo aproximadamente en el intervalo de 0.06 a 0.1 ; en cuanto al lado izquierdo, se puede ver en la tabla 1 que, en las 6 primeras clases o intervalos en los que está dividida la serie, 4 de las esperadas son iguales a cero; por lo que respecta a las otras dos son casi iguales a cero, en tanto que en las frecuencias observadas se puede ver que solamente la primera clase tiene frecuencias de cero.

La distribución observada tiene más observaciones en los extremos que la distribución esperada, lo cual se puede apreciar fácilmente en la tabla 1. Adicionalmente, se puede observar que la gráfica de la distribución observada está sesgada hacia la derecha.

Así, cuestionando el supuesto de independencia y la forma normal de los rendimientos de las acciones bursátiles es como se propone una manera distinta, fractal, de analizar los mercados. En las siguientes secciones se presenta una introducción a este enfoque.

\section{El análisis técnico y los fractales}

El análisis técnico bursátil se ocupa de analizar los precios de los activos, tanto en forma gráfica como a través de indicadores estadísticos y las formaciones que se obtienen con las gráficas de estos precios tienen características fractales. 
Algunas consideraciones sobre el uso de técnicas fractales

en el análisis del mercado accionario bursátil mexicano

En las últimas décadas y, sobre todo, con la amplia disponibilidad de computadoras, se han desarrollado nuevas metodologías para análisis de acciones bursátiles, entre las que se encuentran lo que puede denominarse "técnicas fractales" y que están relacionadas con algunas de las características que definen a estas figuras geométricas.

Aunque no existe una definición estática, sencilla y universalmente aceptada de los objetos denominados "fractales", se puede hacer una aproximación mediante algunas de sus características:

1. Son autosimilares ${ }^{20}$ porque conforme se les observa más de cerca, más detalles muestran y estos acercamientos tienen la misma forma que el objeto completo. En otras palabras, se trata de curvas, superficies o sólidos que son independientes de la escala.

Por ejemplo, para dar una idea intuitiva de la forma fractal de una serie de tiempo como las que se utilizan para analizar los mercados de capitales, en las tres gráficas que aparecen en la figura 2 se muestran los rendimientos (alzas o bajas porcentuales) diarios, semanales y mensuales del Índice de Precios y Cotizaciones de la Bolsa Mexicana de Valores (IPC) para 40 observaciones consecutivas. Si no se tienen las escalas en los ejes, no es posible identificar cuál es cuál: estas imágenes ilustran la autosimilitud en una serie de tiempo.

Figura 2

Gráficas de rendimientos bursátiles del IPC diarios, semanales y mensuales

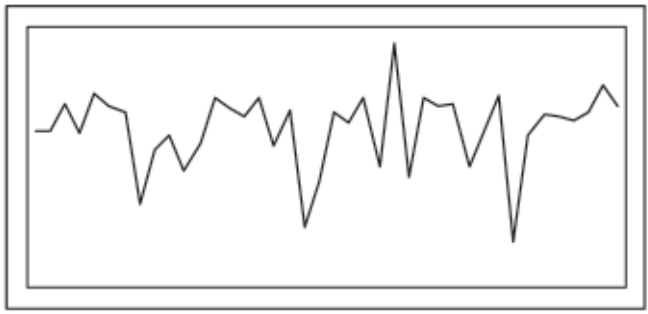

${ }^{20}$ Benoit B. Mandelbrot hace una distinción entre fractales autosimilares y fractales autoafines que es importante para el análisis de precios bursátiles, pero que escapa al alcance de este artículo. Véase Fractals and Scaling in Finance, 1997, pp. 149-151. 

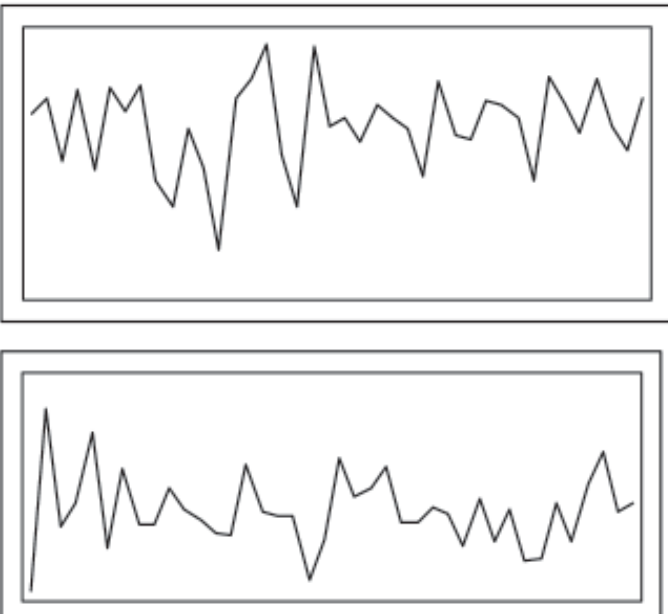

2. Muestran elevada sensibilidad a las condiciones iniciales. Esta característica se puede ilustrar en la construcción de fractales a partir de la iteración de ecuaciones muy simples (como la famosa ecuación logística) en donde se obtienen resultados muy diferentes a partir de valores iniciales muy ligeramente distintos. Determinado valor puede conducir a comportamientos regulares o periódicos o simétricos, en tanto que otro valor, apenas diferente, puede conducir a resultados muy complejos o caóticos.

Para ilustrar lo anterior, conviene recordar que la ecuación logística es de la forma $y_{i+1}=k y_{i}\left(1-y_{i}\right)$, en donde $\mathrm{k}$ es una constante. En la figura 3 se muestra la órbita (los valores sucesivos de la iteración de la ecuación) para $\mathrm{k}=4$ y $y_{\text {inicial }}=0.25$ y en la figura 4 se muestra la órbita correspondiente $\mathrm{a} \mathrm{k}=4 \mathrm{y} \cdot y_{\text {inicial }}=0.25001$.

Figura 3

Gráfica de la ecuación logística para $\mathbf{k}=\mathbf{4} \mathbf{y} y_{\text {inicial }}=0.25$

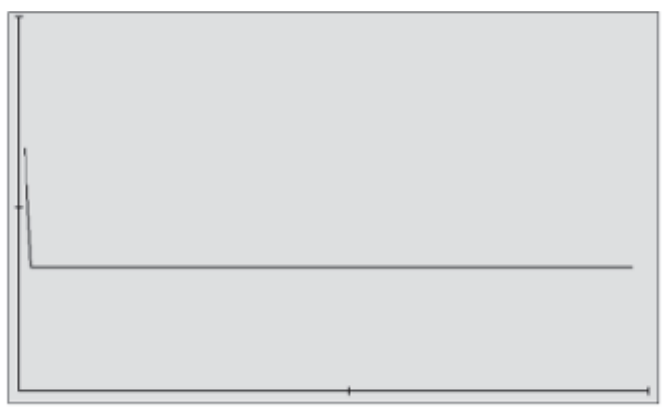


Algunas consideraciones sobre el uso de técnicas fractales

en el análisis del mercado accionario bursátil mexicano

Figura 4

Gráfica de la ecuación logística para $\mathbf{k}=\mathbf{4} \mathbf{y} y_{\text {inicial }}=0.25001$.

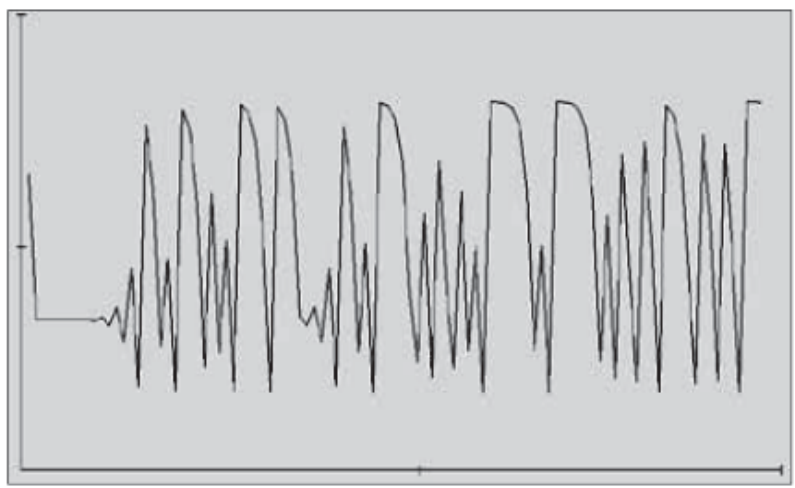

Nótese que una variación de apenas una cienmilésima en el valor inicial hace que la órbita pase de un comportamiento regular (de hecho, un punto fijo igual a 0.75) a un comportamiento muy irregular o caótico que, además, tiene cierto parecido con la forma de las series de rendimientos bursátiles que se ilustran en la figura 1.

3. Tienen dimensión fraccionaria y no entera, como euclideanamente se maneja. Se puede dar una idea intuitiva de esto pensando que una línea tiene dimensión 1, en tanto que una superficie tiene dimensión 2, pero parecería evidente que una curva que se retuerce hasta casi llenar un plano tiene dimensión superior a 1 , pero menor de 2 ; es decir, tiene dimensión fraccionaria o fractal.

En el caso de las series de tiempo que se generan con los precios de valores bursátiles, una inspección visual permite apreciar que comparten las anteriores características de los objetos fractales:

1. Estas series de tiempo pueden dibujarse para diferentes periodos (minutos, horas, días, semanas, etc.) y parecen tener la misma forma. $\mathrm{O}$, en otras palabras, si se observa una gráfica de precios bursátiles que no tiene indicación de la escala, es prácticamente imposible determinar con precisión en qué escala están trazados los valores: son series autosimilares (figura 1). 
2. También muestran un comportamiento que parece conducir a resultados enteramente distintos a partir de condiciones similares. El caso más claro es la ocurrencia de auges marcados o caídas abruptas e inexplicables (comportamiento caótico) cuando en otros periodos con condiciones semejantes el comportamiento resulta ser mucho más regular.

3. Tienen dimensión fractal. Las tradicionales gráficas de barras mediante las que se suelen representar los precios de activos bursátiles y que incluyen datos de apertura, máximo, mínimo y cierre son, en su conjunto, líneas que tienden a llenar el plano.

Estas características de los fractales, con las que coinciden los precios bursátiles, contradicen los supuestos de independencia y de comportamiento normal de los rendimientos bursátiles contenidos en la teoría del paseo aleatorio y en la hipótesis de los mercados eficientes y han dado lugar a la utilización de una medida conocida como “exponente de Hurst" que, como se verá, está relacionada con la dimensión fractal y permite el grado de correlación entre precios bursátiles sucesivos.

Un ejemplo muy interesante de esa estructura fractal de las series de tiempo de precios bursátiles es el planteamiento básico de lo que se conoce como la "Teoría de las olas de Elliott" ${ }^{21}$. Esta teoría propone que el patrón de los precios es regular y que, en su etapa alcista, está formado por cinco olas, tres al alza y dos a la baja en tanto que, en mercados a la baja, se forman las mismas cinco olas, sólo que, en este caso, son tres a la baja y dos al alza. En la figura 5 se ilustra el concepto básico.

A su vez, cada una de las cinco olas consta de otras cinco e igualmente de éstas, tres son en el sentido de la tendencia y dos son de corrección o tendencia contraria. Y, de nuevo, estos submovimientos constan también de olas de menor dimensión y así sucesivamente. Se ilustra en la figura 6 un periodo de alza a largo plazo. La autosimilitud fractal a diversas escalas es evidente en estas dos gráficas.

${ }^{21}$ Frost, Alfred John y Prechter, Robert Rougelot. Elliott Wave Principle,1990. 
Algunas consideraciones sobre el uso de técnicas fractales

en el análisis del mercado accionario bursátil mexicano

Figura 5

El concepto básico de las olas de Elliott

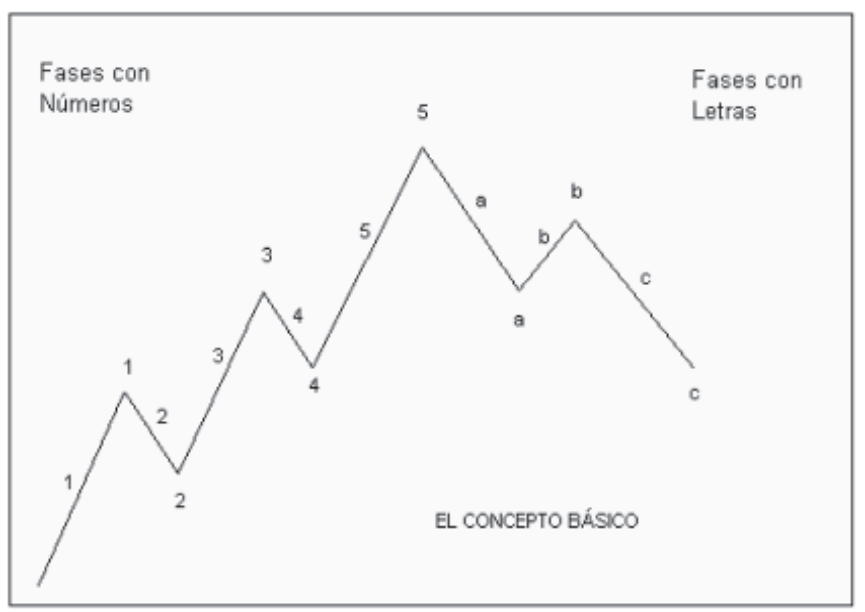

Figura 6

Un periodo de alza a largo plazo, de acuerdo con la teoría de las olas de Elliott

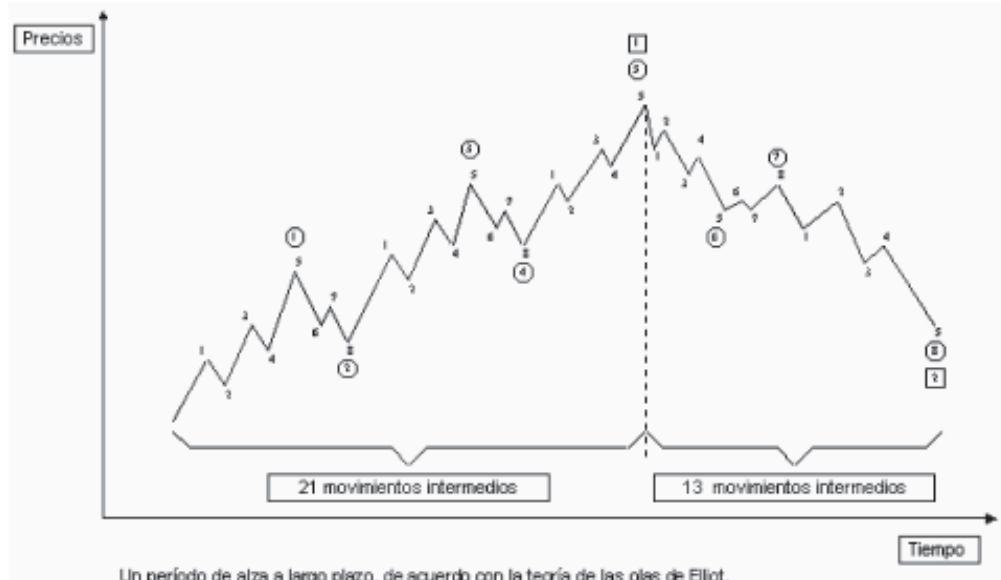

Para dar una idea de los resultados que este sistema de análisis produce, y que no menciona, aunque, como puede apreciarse, sí contiene el concepto de fractal, en ese mismo libro de Frost y Prechter $^{22}$ se pronostica, hacia 1978, año de la publicación

${ }^{22}$ Frost y Prechter, op. cit., p. 176.

No. 224, enero-abril 2008:35-57 
inicial del libro, que el año de 1987 iba a representar una sima importante para el mercado de valores de Nueva York y, en particular, para el Índice Industrial Dow Jones, lo cual efectiva y marcadamente sucedió en octubre de ese año.

\section{El exponente $\mathbf{H}$ de Hurst ${ }^{23}$}

De 1907 a aproximadamente 1947, un hidrólogo de apellido Hurst participó en la construcción de una presa en el río Nilo y estudió el problema del control de la reserva de agua y en su modelo asumió que la parte incontrolable del sistema (la acumulación de agua de lluvia) seguía un recorrido aleatorio porque dependía de muchas variables difíciles de medir. Para evaluar la aleatoriedad de ese sistema hidrológico creó una técnica de análisis a la que denominó "análisis de rangos redimensionados" $(\mathrm{R} / \mathrm{S})$ que consiste básicamente en medir las fluctuaciones (rangos) del nivel de la reserva con respecto a su valor promedio y en dividir esos rangos entre la desviación estándar de las observaciones, con lo que obtuvo una medida que es independiente de la unidad de medición.

Como resultado de sus estudios creó una medida estadística que se conoce como "exponente de Hurst", H, que se utiliza en el análisis de series de tiempo. Con su técnica de R/S, Hurst llegó a la conclusión de que los comportamientos que se suponían aleatorios no lo eran en forma estricta sino que se trataba, más bien, de "recorridos aleatorios sesgados" o, en otras palabras, tendencias con cierto "ruido".

Edgar E. Peters ${ }^{24}$ propone la siguiente adaptación del trabajo de Hurst para extenderlo al estudio de series de tiempo económicas y del mercado de capitales, para ver si estas series son también recorridos aleatorios sesgados y el primer paso consiste en definir primero un rango que sea comparable a las fluctuaciones de los niveles para conformar la siguiente serie de tiempo:

$$
X_{t, n}=\sum_{u=1}^{t}\left(e_{u}-M_{N}\right)
$$

donde:

$X_{t, n}=$ desviación acumulada en N periodos.

$e_{u}=$ flujo de entrada en el año $\mathrm{u}$.

$M_{N}=$ promedio de $e_{u}$ para $\mathrm{N}$ periodos.

\footnotetext{
${ }^{23}$ Adaptado de Peters, op.cit., 1991.

${ }^{24}$ Ibídem, 1991, p. 63.
} 
Algunas consideraciones sobre el uso de técnicas fractales

en el análisis del mercado accionario bursátil mexicano

Después, se calcula el rango como la diferencia entre los niveles máximo y mínimo alcanzados en la fórmula anterior:

$$
R=\operatorname{Max}\left(X_{t, n}\right)-\operatorname{Min}\left(X_{t, n}\right)
$$

Para comparar distintos tipos de series de tiempo, Hurst dividió este rango entre la desviación estándar de las observaciones originales (como se menciona arriba, esta división entre la desviación estándar produce rangos que son independientes de la unidad de medición, lo cual permite hacer comparaciones válidas entre series de tiempo con variables dadas en diferentes unidades de medición). Asumiendo que ese rango redimensionado debe aumentar con el tiempo, Hurst planteó la siguiente relación:

$$
R / S=(a N)^{H}
$$

donde:

$\mathrm{R} / \mathrm{S}=$ rango redimensionado

$\mathrm{N}=$ número de observaciones

$\mathrm{a}=$ una constante

$\mathrm{H}=\mathrm{el}$ exponente de Hurst

"Según la mecánica estadística, $\mathrm{H}$ debe ser igual a 0.5 si la serie es un recorrido aleatorio" "25. Cuando Hurst aplicó su estadístico al historial de descargas del río Nilo y a otros fenómenos naturales encontró que $\mathrm{H}$ era normalmente mayor de 0.5 , lo cual implicaba que las observaciones no eran independientes, sino que cada evento acarreaba una "memoria" finita, pero de largo plazo, de todos los eventos precedentes. Otra manera de interpretar esto es considerar que la serie es una cadena de eventos interconectados. Peters propone expresar como correlación el impacto del presente sobre el futuro de la siguiente manera:

$$
C=2^{(2 H-1)}-1
$$

donde:

$\mathrm{C}=$ medida de la correlación

$\mathrm{H}=$ exponente de Hurst

${ }^{25}$ Peters, 1991, p. 63 
El exponente de Hurst permite clasificar a las series de tiempo en tres categorías, las cuales se revisan más adelante y resulta ser una medida estadística muy robusta porque hace pocas suposiciones sobre el sistema que se estudia y, en particular, permite clasificar las series sin importar cuál sea la forma de la distribución subyacente. Además, el exponente de Hurst $(\mathrm{H})$ describe la probabilidad de ocurrencia de dos eventos consecutivos. Si H $=0.6$ hay, en esencia, una probabilidad de $60 \%$ de que, si el último movimiento fue positivo, el siguiente también lo sea. Las tres categorías en que se clasifican las series de tiempo de acuerdo con $\mathrm{H}$ son a) $\mathrm{H}=0.5, \mathrm{~b}) 0 \leq \mathrm{H}<$ 0.5 , y c) $0.5<\mathrm{H}<1$.

a) Cuando la serie de tiempo arroja $\mathrm{H}=0.5$, se tiene una serie aleatoria. Las observaciones no están correlacionadas y

$$
\begin{aligned}
& C=2^{(2 H-1)}-1=0, \text { ya que } \\
& 2^{2 H-1}=1 \\
& (2 H-1) \log 2=\log 1 \\
& 2 H-1=\frac{\log 1}{\log 2}=\frac{0}{0.30103}=0 \\
& 2 H=1 \\
& H=0.5
\end{aligned}
$$

b) Cuando $0 \leq \mathrm{H}<0.5$ se tienen series antipersistentes o ergódicas, a las que con frecuencia se denomina "de reversión de la media". La característica de este tipo de series es que, si el sistema ha estado arriba en el periodo anterior, es más probable que esté abajo en el siguiente, y viceversa.

c) Cuando $0.5<\mathrm{H}<1.0$ se está ante una serie persistente, que refuerza la tendencia. Si la serie estaba arriba (o abajo) en el periodo previo, lo más probable es que siga arriba (o abajo) en el periodo siguiente. Hurst caracterizó a este tipo de series persistentes como "recorridos aleatorios sesgados". Por un lado, la fuerza del sesgo depende de qué tan lejos de 0.5 se encuentra $\mathrm{H}$ y, por otro, la fuerza de la conducta de reforzamiento de la tendencia aumenta conforme más se aproxima $\mathrm{H}$ a 1, porque esto implica que la correlación tiende al 100\%.

Por otro lado, los mercados financieros, que tienen evidentemente mecanismos de retroalimentación dependientes del tiempo (observaciones correlacionadas), son sistemas dinámicos no lineales cuyas principales características son: 
Algunas consideraciones sobre el uso de técnicas fractales

en el análisis del mercado accionario bursátil mexicano

- El efecto de retroalimentación produce correlaciones y tendencias a largo plazo; en otras palabras las series de tiempo de los rendimientos bursátiles son persistentes debido a que $0.5<\mathrm{H}<1$.

- Los mercados son erráticos (llegan a niveles críticos) bajo ciertas condiciones $\mathrm{y}$ en ciertos momentos.

- Se producen series de tiempo de rendimientos que, a menores incrementos de tiempo, siguen teniendo el mismo aspecto y tienen características estadísticas similares (estructura fractal).

- Es menos factible hacer pronósticos conforme más adelante en el futuro se observa porque hay una sensible dependencia de las condiciones iniciales.

Para describir la naturaleza fractal de $\mathrm{H}$, el exponente de Hurst, Peters menciona que Mandelbrot demostró que el inverso de $\mathrm{H}$ es la dimensión fractal, por lo que una serie verdaderamente aleatoria sería bidimensional y llenaría un plano, porque $D=\frac{1}{H}=\frac{1}{0.5}=2$. Y, como las series de tiempo que reúnen los rendimientos de instrumentos bursátiles arrojan valores de $\mathrm{H}$ que son mayores de 0.5 y menores de 1 , sus dimensiones fractales son cantidades mayores que $1 \mathrm{y}$ menores que 2 .

Para evaluar los conceptos anteriores en el mercado mexicano, se estimaron los coeficientes de Hurst para el IPC y para las acciones Bimbo A, Femsa UBD, Kimber A y Telmex L, que son acciones para las que se obtuvieron precios de mercado desde el primero de abril de 1987 y hasta el 26 de junio de 2006.

Utilizando el paquete de computación AutoSignal, versión 1.7, que comercializa la empresa estadunidense Systat, Inc., se estimaron los coeficientes de Hurst (H) de estas cinco series de precios utilizando los rendimientos según lo propone Peters, como el precio del día entre el precio del día anterior o, en símbolos, $r=\frac{P_{i}}{P_{i-1}}$
Se muestran los resultados en la tabla 2 .

Tabla 2

Coeficientes H para cuatro acciones y el IPC de la BMV

\begin{tabular}{|l|l|}
\hline \multicolumn{1}{|c|}{ Acción o Índice } & \multicolumn{1}{c|}{ H } \\
\hline Bimbo A & 0.567324 \\
\hline Femsa UBD & 0.506572 \\
\hline IPC & 0.591315 \\
\hline Kimber A & 0.55756 \\
\hline Telmex L & 0.52926 \\
\hline
\end{tabular}


Como puede verse, los coeficientes $\mathrm{H}$ de las cuatro acciones y del IPC son, todos, superiores, aunque cercanos, a 0.5 , lo cual podría ser señal de que se trata de series persistentes lo cual indica, a su vez, que existe correlación entre los rendimientos sucesivos, por lo que es más probable que el movimiento siguiente sea en el mismo sentido que el anterior a que lo sea en el sentido contrario. Esto, a su vez, es una característica que parecería mostrar la posibilidad de encontrar mecanismos para hacer pronósticos del comportamiento de los precios, cuando menos a corto plazo. Y esto último es precisamente, lo que el análisis técnico bursátil pretende hacer y es un objetivo al que, también posiblemente, el análisis fractal pudiera coadyuvar.

Sin embargo, es importante señalar aquí varias consideraciones:

1. La primera de ellas es que la forma en que Peters propone calcular rendimientos, como cociente entre precios, no arroja precisamente rendimientos sino, más bien, montos. Sin embargo, a pesar de la inexactitud de llamar "rendimientos" a lo que formalmente se conoce como "monto" en matemáticas financieras, tal como se ve enseguida, utilizar los rendimientos mismos no produce diferencias en los valores calculados de $\mathrm{H}$.

Así, en vista de lo anterior, se estimaron los coeficientes Hurst para rendimientos del IPC y de las cuatro acciones mexicanas, que se calculan como $i=\frac{P_{i}}{P_{i-1}}-1$, y se
obtuvieron los mismos resultados anteriores.

2. Otra consideración es la que se refiere a la realización de pruebas sobre la validez de los resultados, sobre lo cual Peters propone revolver los datos de manera que el orden de las observaciones sea completamente diferente al de la serie de tiempo original. Como las observaciones siguen siendo las mismas, su distribución de frecuencias sigue siendo la misma y, para hacer la prueba, se vuelve a calcular el exponente $\mathrm{H}$ con los datos revueltos:

Si el conjunto de observaciones es realmente independiente, entonces $\mathrm{H}$ debe mantenerse prácticamente sin cambio porque no hay correlación entre ellas, es decir, porque no existen efectos de memoria de largo plazo y, si es así, revolver los datos no tendría efecto sobre los aspectos cualitativos de los datos. Pero, si existe el efecto de memoria de largo plazo, al revolver los datos se destruye la estructura del sistema $\mathrm{y}$, por ello, el $\mathrm{H}$ estimado debe ser mucho menor y más cercano a 0.5 aun cuando la distribución de frecuencias de las observaciones permanece sin cambios.

Se llevó a cabo esta prueba asignando números aleatorios a cada uno de los precios, ordenados originalmente como serie de tiempo y, después, se reordenó la serie de 
Algunas consideraciones sobre el uso de técnicas fractales

en el análisis del mercado accionario bursátil mexicano

acuerdo con esos números aleatorios y se volvieron a calcular los coeficientes $\mathrm{H}$. Se muestran ambos conjuntos de resultados en la tabla 3.

Tabla 3

Coeficientes $\mathrm{H}$ para la serie de tiempo original y para los mismos datos ordenados aleatoriamente

\begin{tabular}{|c|c|c|}
\hline Acción o Índice & $\begin{array}{c}\text { H de } \\
\text { rendimientos }\end{array}$ & $\begin{array}{c}\text { H con observaciones } \\
\text { con orden aleatorio }\end{array}$ \\
\hline Bimbo A & 0.567324 & 0.567324 \\
\hline Femsa UBD & 0.506572 & 0.506572 \\
\hline IPC & 0.591315 & 0.591671 \\
\hline Kimber A & 0.55756 & 0.55756 \\
\hline Telmex L & 0.52926 & 0.570557 \\
\hline \multicolumn{2}{|r}{} \\
\hline
\end{tabular}

Estos resultados muestran que, como los coeficientes $\mathrm{H}$ prácticamente no sufrieron cambio al ordenar aleatoriamente las observaciones, entonces se contradice la conclusión que se esbozó antes en el sentido de que puede pensar que se tiene un conjunto de datos con memoria de largo plazo; este último ejercicio parecería indicar lo contrario.

Este resultado parecería indicar la necesidad de ampliar el análisis de las técnicas para estimar $\mathrm{H}$, o de usar un mayor número de valores bursátiles, o de utilizar bases de datos más amplias, que pudieran conducir a resultados más concluyentes.

\section{Comentarios finales}

La conclusión preliminar más importante que se puede extraer de estos análisis es que el análisis de rangos redimensionados, creado por Hurst, y según propone Peters aplicarlo a los mercados financieros, ofrece evidencia adicional, aunque contradictoria, de que la hipótesis de los mercados eficientes y la teoría del paseo aleatorio tienen serias deficiencias porque parten de los supuestos de que los rendimientos sucesivos se distribuyen en forma normal y que son independientes entre sí y no parece ser éste el caso.

Por otro lado, tratándose aquí, como se ha mencionado repetidamente, de un análisis exploratorio de estas técnicas fractales que se encuentra en sus inicios, es mucho lo que falta por hacer para llegar al objetivo principal de crear mecanismos que permitan mejorar los pronósticos del comportamiento de los precios de los valores bursátiles

En otras palabras, existe toda una serie de análisis que aún falta por llevar a cabo para tratar de ver si es posible encontrar mecanismos fractales, es decir, de sistemas dinámicos no lineales, esto es, de teoría del caos, que permitan hacer pronósticos de precios bursátiles consistentemente superiores a los del promedio. 


\section{Bibliografía}

BAChelier, Louis, Théorie de la spéculation, Annales Scientikfiques de l'Ecole Normale Superieur, 3a. serie, tomo 17, 1900.

Buffet, Warren E., "The Superinvestors of Gram.-and-Doddsville", Hermes, revista de la Columbia Business School, www.sandmansplace.com/Superinvestors_Graham_Dodd.html, 1984.

DíAz Mata, Alfredo, "Caos, fractales, mercados de valores y triceversa", Contaduría y Administración, Facultad de Contaduría y Administración, Universidad Nacional Autónoma de México, núm. 181, abril-junio, México, 1996.

, Un sistema computarizado para análisis de acciones bursátiles en México, tesis para obtener el grado de Maestro en Finanzas, Facultad de Contaduría y Administración, Universidad Nacional Autónoma de México, 2002.

- Un sistema computarizado para análisis de acciones bursátiles en México, tesis para obtener el grado de Doctor en Ciencias de la Administración, Facultad de Contaduría y Administración, Universidad Nacional Autónoma de México, 2005.

FAma, Eugene F., "The Behavior of Stock Market Prices”, Journal of Business, núm. 38, EUA, enero, 1965.

, Foundations of Finance, Basic Books, Inc., EUA, 1976.

Frost, Alfred John y Prechter, Robert Rougelot, Elliott Wave Principle, 6a. edición ampliada, EUA, 1990.

López Herrera, Francisco, "Análisis de la eficiencia del mercado accionario mexicano", Contaduría y Administración, Facultad de Contaduría y Administración, Universidad Nacional Autónoma de México, núm. 191, octubre- diciembre, México, 1998.

Malkiel, Burton G., Un paseo aleatorio por Wall Street, Alianza Editorial, España, 1992.

Mandelbrot, Benoit B., Fractals and Scaling in Finance, Springer, EUA, 1997. va York, 1977, 1982, 1983.

Peters, Edgar E., Chaos and Order in the Capital Markets, John Wiley \& Sons, Inc. EUA, 1991. 
Algunas consideraciones sobre el uso de técnicas fractales

en el análisis del mercado accionario bursátil mexicano

-, Fractal Market Analysis. John Wiley \& Sons, Inc., EUA, 1994.

Ross, Stephen A., Westerfield, Randolph W. y Jordan, Bradford D., Finanzas corporativas, Irwin, 3a edición, España, 1995.

Internet

www.finsat.com

www.investorhome.com/anomaly.htm

www.investorhome.com/anomtec.htm

www.sandmansplace.com/Superinvestors_Graham_Dodd.htm.1

www.stochastik.uni_freiburg.de/bfsweb/LBachelier/mafi092XX.pdf

www.systat.com/downloads/?sec=d005 (A) 